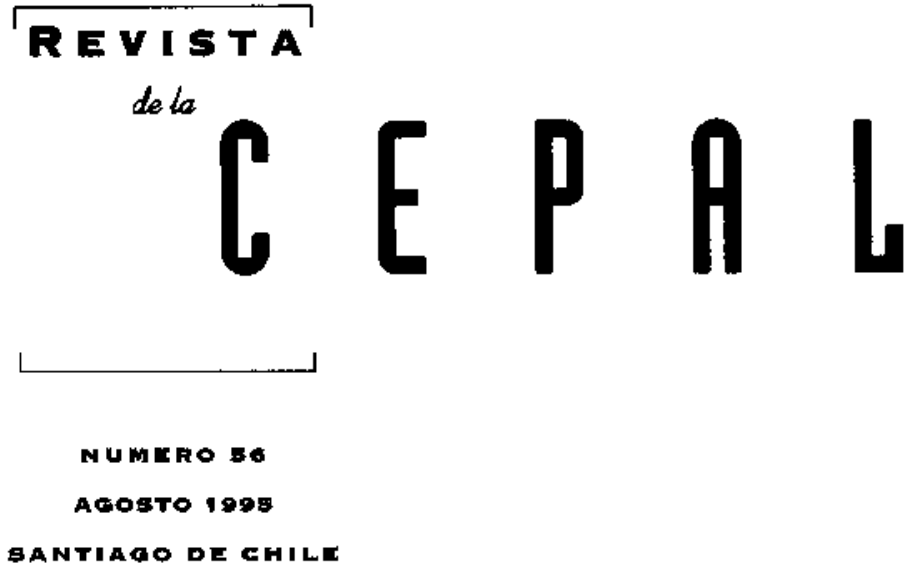

ANIBAL PINTO

Director

EUGENIO LAHERA

Secretario Técnico

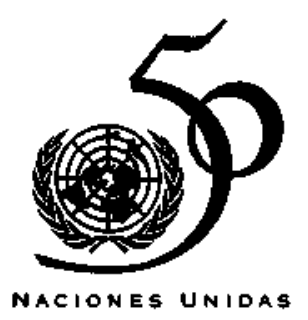


Democracla y desarrollo

Fernando H. Cardoso

¿Es posible crecer con equidad?

Joseph Ramos

Estabilidad y estructura: Interacclones en el crecimiento económlco

Jose Maria Fanelli y Roberto Frenkel

Reforma a los sistemas de pensiones en América Latina

Andras Uthoff

Tendenclas económicas en China: significado para el comerclo con América Latina y el Caribe

Mikio Kuwayama

El Intercamblo económico entre América Latina y las

economias dinámicas de Asla

Ronald Sprout

La relación económica entre la Amórica Latina y la Unlón Europea

Roberto Smith Perera

Nuevas implicaclones de las reglas do orlgen

Eduardo Gitli

Globalización y reestructuración energética en Amórlca Latina

Fernando Sánchez Albavera

El caloidoscoplo de la competitlvidad

Geraldo Muller

La privatización de los servicios públicos del agua

Miguel Solanes

¿Cuénto se puede gastar en educaclón?

Guillermo Labarca

Mujeres y migranteo: deslgualdades en el

mercado laboral de Santlago de Chile

Ivonne Szasz

Orlentaciones para los colaboradores de la Rovista de la CEPAL 


\section{Mujeres y migrantes: desigualdades en el mercado laboral de Santiago de Chile.}

\section{Ivonne Szasz}

Profesora-investigadora, Centro de Estudios Demográficos y de Desarrollo Urbano de El Colegio de México
Las políticas sociales que buscan reducir las inequidades en el mercado de trabajo deben sustentarse en conocimientos sólidos sobre los elementos que generan condiciones desventajosas para segmentos específicos de esos mercados. El presente artjculo describe desigualdades en la estructura ocupacional y en los ingresos que afectan a las mujeres económicamente activas y en particular a las inmigrantes económicamente activas en Santiago de Chile, y aporta algunos elementos para comprender los factores que determinan esas desventajas. La evolución económica chilena ha sido considerada un ejemplo para los demás páses latinoamericanos que se abren hacia los mercados internacionales. Sin embargo, es preciso evitar una mayor concentración de la riqueza, la persistencia de los niveles de pobreza, el aumento de la heterogeneidad laboral y las desigualdades en los ingresos del trabajo. Este artículo busca contribuir a develar las causas de las condiciones desventajosas en que funcionan algunos segmentos del mercado de trabajo urbano en Chile. La elevada escolaridad de las mujeres y de las inmigrantes economicamente activas en Santiago contrasta con su distribución ocupacional y sus ingresos. Mejorar las calificaciones de la fuerza de trabajo no sera suficiente para reducir las inequidades en los mercados de trabajo latinoamericanos. Aquí se postula que se requiere conocer y enfrentar también las construcciones de género que condicionan la estructura de estos mercados laborales, generando discriminaciones ocupacionales y salariales que no parecen vinculadas con la capacitación de los trabajadores. 


\section{I \\ Introducción}

Las medidas de reconversión económica que los demás parses latinoamericanos iniciaron hacia fines de los años ochenta se aplicaron en Chile en 1975, de modo que sus efectos pudieron observarse en el mercado de trabajo urbano en el decenio siguiente.

La evolución económica chilena suele considerarse un ejemplo átil para los demás países de la región, por sus logros macroeconómicos. Sin embargo, también suele expresarse preocupación por el aumento de la desigualdad en la distribución del ingreso y la precariedad de algunos segmentos del mercado de trabajo, que pueden explicar las elevadas proporciones de pobreza urbana. Las predicciones son optimistas en cuanto a que el crecimiento sostenido puede mejorar los ingresos y las condiciones de vida de los sectores de mayor productividad y que es posible derivar recursos hacia los sectores menos favorecidos a través de políticas sociales, las que al mismo tiempo deben intentar elevar su capacitación y mejorar su inserción productiva (Altimir, 1994; CEPAL, 1992). Para llevar adelante este tipo de políticas tendientes a una mayor equidad se necesita conocer los factores que determinan la inserción desventajosa de algunos grupos en el mercado de trabajo.

Este artículo explora la situación de dos sectores de ese mercado en Santiago de Chile que se encuentran en condiciones de desventaja relativa - las mujeres económicamente activas y las mujeres inmigrantes- y pone de relieve algunos aportes de la perspectiva de género al análisis de la participación económica y la migración femeninas que permiten contribuir al diseño de políticas públicas encaminadas a hacer más equitativas las condiciones de trabajo y de vida de la poblacion latinoamericana.

La investigación que dió origen al presente artículo analizo la evolución del mercado de trabajo femenino en una de las metrópolis de America Latina - la ciudad de Santiago de Chile-- y las características de un segmento específico de ese mercado: el de las mujeres inmigrantes integradas en la actividad económica. Se realizó en el Centro Latinoamericano de Demografía (CELADE) en 1991-1992 con el patrocinio de la Fundación Rockefeller, usando fuentes documentales, bibliográficas y estadísticas. Su objetivo fue analizar los cambios en la estructura del mercado de trabajo femenino de Santiago y en la inmigración de mujeres hacia esa ciudad entre 1950 y 1990, así como la inserción laboral de las mujeres inmigrantes entre 1960 y 1982 . Las fuentes utilizadas para el análisis de los mercados de trabajo fueron los censos de población y la Encuesta Nacional de Empleo del Sistema Integrado de Encuestas en Hogares. Los datos sobre las mujeres inmigrantes proceden de la encuesta sobre migración al Gran Santiago efectuada por el CELADE en 1962 y de muestras de los censos de población de 1970 y 1982 elaboradas por CELADE, correspondientes a lo que era la provincia de Santiago y es hoy la Región Metropolitana. La información que se menciona en el presente artículo corresponde a esas fuentes.

II

\section{Los cambios económicos y el mercado de trabajo en Chile en los años ochenta}

Los grandes cambios socioeconómicos ocurridos en América Latina y en Chile a raíz de la crisis econ6mica de comienzos de los af́os ochenta, seguida de las políticas de ajuste, de la reconversión productiva y de la apertura a los mercados internacionales, tuvieron distintos efectos en diferentes segmentos del mer- cado de trabajo. Diversos estudios coinciden en señalar que aumentó la heterogeneidad laboral, se acentuaron las diferencias entre los ingresos de diversos sectores de trabajadores y hubo procesos de precarización en partes del mercado de trabajo (PREALC, 1991; CEPAL, 1994; García, 1993). 
En especial, experimentó cambios el mercado de trabajo femenino. La participación de las mujeres en la actividad economica se había acrecentado sostenidamente desde mediados de los años cincuenta, debido a los procesos de urbanización, la expansión de los servicios personales, sociales y comunitarios, y el paulatino aumento de la escolaridad de las mujeres. A partir de la crisis de los años ochenta se registró un aumento mayor de la participación económica femenina. Este comportamiento se ha considerado anticíclico, por la mayor incorporación de mujeres en las coyunturas recesivas y por lo mal remunerado o precario de muchas de sus actividades (CEPAL, 1994; Pollack, 1990).

En la década de los ochenta también hubo cambios en la edad y estado civil de las mujeres económicamente activas, registrándose un incremento de aquéllas casadas y con hijos, y un aumento de las ocupaciones no asalariadas.

El caso de Chile ha llamado la atención de los estudiosos del tema por el inicio temprano de las políticas de apertura hacia los mercados externos y de la desregulación laboral, por la gravedad de los efectos de la recesión y de las políticas de ajuste en los mercados de trabajo urbanos en un contexto político autoritario, por los éxitos macroeconómicos del posterior proceso de consolidación de la transformación productiva, pero también por la mayor desigualdad en la distribución del ingreso, las elevadas proporciones de población pobre y la precarización de segmentos de su mercado de trabajo (García, 1991 y 1993; Altimir, 1994).

A continuación complementaremos estos análisis globales del mercado de trabajo chileno con un examen de la evolución de la fuerza de trabajo femenina y de las características particulares de las mujeres inmigrantes, con la finalidad de contribuír a identificar segmentos vulnerables del mercado de trabajo metropolitano, así como señalar algunos elementos que contribuyen a esa vulnerabilidad.

\section{III}

\section{Las migraciones femeninas hacia las grandes ciudades latinoamericanas}

Desde el inicio del proceso de industrialización sustitutiva de las importaciones, en América Latina existí un claro predominio femenino en los desplazamientos migratorios internos. Esta primacía fue más marcada en las corrientes rural-urbanas y en las migraciones que tuvieron como lugar de destino a las ciudades principales.

Los factores que condicionaron el éxodo rural en la región afectaron de manera selectiva a la población femenina, transformando de manera preferente a las mujeres rurales jóvenes en mano de obra excedente. A su vez, la segregación por sexo y condición migratoria en los mercados de trabajo configuro una demanda particular de trabajadoras inmigrantes en las grandes urbes. En las metrópolis de America Latina, las migrantes se han concentrado en un pequeño número de ocupaciones reservadas a las mujeres, y se han agrupado, más que las no migrantes, en el sector terciario y en actividades manuales, en especial en el servicio doméstico (Pardo, 1987; De Oliveira, 1984; Elton, 1978; Raczynski y Vergara, 1979; Elizaga, 1970; Recchini de Lattes, 1990).
Las investigaciones actuales sobre la migración interna en América Latina examinan las repercusiones de la internacionalización de las economías sobre los movimientos migratorios, así como los cambios en la inserción laboral de distintos miembros de los grupos familiares. Algunos análisis agregan nuevas dimensiones para la comprensión de los procesos migratorios, como las fuerzas culturales, ideológicas y políticas, que operan con una dinámica compleja diferente de la económica (Lim, 1989; De Oliveira, 1984; Lattes, 1989). Una de esas dimensiones socioculturales es el papel de las mujeres en la sociedad. La segregación por sexo y por condición migratoria en los mercados de trabajo expresa valores y prácticas que restringen a las mujeres a su rol reproductivo en el ámbito privado, o a su inserción en labores que constituyen una extensión de ese rol en el ámbito económico y que se encuentran socialmente desvalorizadas. La menor valoración de las labores desempeñadas por mujeres delinea las particularidades de la migración femenina y de la inserción ocupacional de las mujeres (García de Fanelli, 1989; Muñoz, 1988; Arriagada, 1990; Hugo, 1991). 
Otras dimensiones culturales, como el control social sobre la sexualidad femenina, definen rasgos particulares para las migraciones de mujeres. Ellas deben trasladarse, emplearse y residir en condiciones que no permitan cuestionar el recato de las jovenes solteras y la fidelidad de las mujeres casadas. Los traslados de mujeres con motivaciones laborales se vinculan con el desarrollo de segmentos específicos del mercado de trabajo que son socialmente aceptables para las mujeres. La posición en la familia, la distribución de tareas en el hogar, los patrones de autoridad intrafamiliar y las pautas de nupcialidad tienen mayor importancia en el estudio de las migraciones femeninas que en las masculinas (Hugo, 1991; De Oliveira, 1984).

En los últimos años, las migraciones intemas en la región ban crecido en variedad y complejidad. A los factores tradicionales de la migración de mujeres hacia las grandes ciudades se agregaron el aumento de la pobreza urbana y la mayor actividad económica de mujeres no migrantes, los procesos de urbanización del campo y de asalariamiento de la población rural, la destegulación de las condiciones de trabajo y el creciente empleo de mujeres de escasos recursos en la agroindustria y la industria maquiladora de exportación. Estas transformaciones, junto con el aumento de la escolaridad femenina y el descenso de la fecundidad, alteraron las pautas tradicionales de migración femenina y dieron origen a nuevas formas de desplazamiento. Disminuyo el predominio de mujeres y la proporción de adolescentes en la migración a las grandes ciudades y se incrementaron la movilidad temporal, la migración interurbana, los traslados cotidianos, la residencia urbana con empleo rural y la migración de origen y destino rural.

A pesar de los rasgos claramente diferenciados de la migración femenina, muy pocas investigaciones describen por separado sus características (Elizaga, 1970; Elton, 1978; Raczynski y Vergara, 1979; De Oliveira, 1984). La carencia es más marcada si nos referimos a las particularidades que la desigualdad social entre los géneros introduce en la migración femenina y en la inserción laboral de las mujeres. Con excepción de algunos estudios de casos realizados en los últimos años, la dimensión de género no constituye un elemento conceptual incorporado al análisis de las migraciones, y esta ausencia ha afectado el desarrollo de políticas y programas destinados a mejorar la situación social de las mujeres latinoamericanas.

En nuestra investigación incorporamos el análisis de algunos aspectos de las desigualdades de género. El examen de uno de esos aspectos, el de las causas de la emigración femenina rural en Chile durante el proceso de industrialización sustitutiva de importaciones, indica que existen causas estructurales y motivaciones personales diferentes para las migraciones femeninas y masculinas (Aranda, 1982; Pardo, 1987; Raczinsky y Vergara, 1979; PREALC, 1979).

Basándonos en fuentes estadísticas, analizamos otros aspectos de la desigualdad entre los géneros, que se exponen en el presente artículo. Uno de ellos es el de las diferencias en la concentración ocupacional y los ingresos por trabajo entre los hombres y mujeres de la zona metropolitana de Santiago en 1990 . Otro se relaciona con las desigualdades en la estructura ocupacional y de ingresos observada en 1982 en la provincia de Santiago entre las mujeres inmigrantes econónicamente activas, por un lado, y los varones inmigrantes y mujeres no migrantes, por otro. Esta comparación nos permitio aventurar algunas interpretaciones de las desventajas de las migrantes fundadas en dimensiones de género, que permitirán orientar políticas sociales dirigidas a estos grupos de población.

\section{IV}

\section{El mercado de trabajo femenino en}

\section{la ciudad de Santiago}

El desarrollo de segmentos específicamente femeninos de los mercados de trabajo en los lugares de origen y destino de las migraciones aparece condicionando el volumen y las características de los movimientos femeninos, así como las posibilidades de inserción ocupacional de las migrantes. En el caso de Chile, la selectividad femenina ha sido más marcada en las migraciones de origen rural y semimural que tienen como lugar de destino la ciudad de Santiago (Martínez, 1990; Raczynski y Vergara, 1979; He- 
rold, 1979). Aunque en los últimos años ha disminuido la tasa de crecimiento de la migración de mujeres hacia esta ciudad, probablemente por la expansión del empleo femenino en la agroindustria, su volumen sigue siendo creciente y continúa el predominio femenino entre los migrantes que proceden de regiones con proporciones importantes de población rural (Martínez, 1990).

Las profundas transformaciones sufridas por los mercados de trabajo chilenos en los últimos veinte años pueden estar afectando tanto los patrones migratorios como la insercion ocupacional de los inmigrantes. Por su importancia como lugar de destino preferente de la migración de mujeres de origen rural, examinaremos en seguida la evolución del mercado de trabajo femenino en Santiago entre 1952 y 1990 , para luego analizar la fuerza de atracción que ejerce ese mercado sobre las migrantes, las características de ellas y las condiciones en que se integran en la actividad económica de la ciudad.

En 1950, la tasa de participación económica femenina en la capital de Chile era considerablemente más alta que en otras ciudades latinoamericanas: alcanzaba a un tercio de las mujeres de 12 años y más. La participación económica se concentraba en las mujeres solteras, y era más alta antes de los 25 años. La mayor parte de estas mujeres económicamente activas tenían menos de siete años de escolaridad. Más de dos tercios se ocupaban en el sector terciario, especialmente en los servicios personales y del hogar (principalmente trabajadoras manuales dedicadas al servicio doméstico y otros servicios personales) y una cuarta parte eran operarias y artesanas calificadas (modistas, costureras y tejedoras).

En 1970, la tasa de participación había disminuido de un tercio a una cuarta parte de las mujeres, habiendo mermado sobre todo la participación de jovencitas menores de $\mathbf{2 0}$ afios. Las mujeres económicamente activas con menos de siete años de escolaridad alcanzaban apenas al 35\%. La proporción de mujeres en los servicios personales y del hogar había disminuido y la superaban las mujeres en servicios sociales y comunitarios y de apoyo a la producción. Las trabajadoras manuales disminuyeron proporcionalmente, especialmente las no calificadas, y el aumento de trabajadoras no manuales se registro sobre todo en el grupo de oficinistas (Szasz, 1994).

Esta evolución, que aparece como una consecuencia del aumento de la escolaridad de la población femenina y de una disminución en la participación económica de las mujeres de sectores populares, fue modificada ligeramente por los cambios macroeconómicos que experimentó el país entre 1970 y 1990 . En este último año se registraba una participación econ6mica mayoritaria de mujeres casadas en edades reproductivas. Antes de 1970 , el $60 \%$ de las mujeres económicamente activas eran solteras, mientras que en 1990 las solteras habían descendido al $40 \%$ de las trabajadoras. Hasta 1970, las edades de mayor participación económica estaban por debajo de los 25 afios, mientras que en 1990 los mayores niveles se daban entre los 25 y los 45 años (Szasz, 1994).

En 1990, la mitad de las mujeres trabajadoras tenía más de 12 años de escolaridad y apenas una cuarta parte tenía menos de 7 años de instrucción escolar. Se concentraban en proporciones menores que en 1970 en los servicios personales y del hogar. Cerca de un $40 \%$ se dedicaba a actividades no manuales. Además de aumentar las oficinistas, se incrementaron las profesionales, técnicas y directivas. Las principales diferencias con la evolución anterior se observan en la edad y estado conyugal de las mujeres economicamente activas, el aumento de las tasas de desempleo femenino, sobre todo en los sectores de menores ingresos, y el tipo de ocupaciones que elevaron su dinamismo en la metrópoli. En el período 1982-1990 se observó, además del aumento de trabajadoras no manuales, un incremento de las trabajadoras manuales no calificadas (obreras, jomaleras, sirvientas) y de las vendedoras dependientes y ambulantes. $Y$ aunque en este período aumentaron las mujeres asalariadas, mucho más lo hicieron las trabajadoras por cuenta propia y las trabajadoras familiares no remuneradas (Szasz, 1994).

La ocupación que experimento mayores cambios en este último período fue el servicio doméstico. Esta labor agrupaba a la tercera parte de las mujeres económicamente activas en los años cincuenta, y cuarenta años después absorbía algo más de la cuarta parte de las trabajadoras. A pesar de su menor importancia en el conjunto, en 1990 la mitad de las trabajadoras manuales continuaban siendo sirvientas. Su número se incrementó considerablemente, y su tasa de crecimiento medio anual alcanzó los niveles más elevados en el período 1982-1990.

El cambio más notable que experimentó este grupo de ocupación fue el aumento de las trabajadoras domésticas que no viven en el lugar de trabajo. Las empleadas domésticas no residentes representaban el $11 \%$ del total en 1952, mientras que en 1990 la mayoría de las personas en esa ocupación eran no residentes (cuadro 1). 
CUADRO 1

Santiago de Chile: Proporción de mujeres en - at arviclo dombatico con y sin realdoncla on el lugar de trabajo, entre 1957 y 1990

\begin{tabular}{lccc}
\hline & $\begin{array}{c}\text { Residentes } \\
\text { en el lugar } \\
\text { de trabajo }\end{array}$ & $\begin{array}{c}\text { No residentes } \\
\text { en el lugar } \\
\text { de trabajo }\end{array}$ & Total \\
\hline 1957 & 88.9 & 11.1 & 100.0 \\
1967 & 85.4 & 14.6 & 100.0 \\
1972 & 85.7 & 14.3 & 100.0 \\
1974 & 64.7 & 35.3 & 100.0 \\
1975 & 64.9 & 35.1 & 100.0 \\
1976 & 58.3 & 41.7 & 100.0 \\
1977 & 57.3 & 42.7 & 100.0 \\
1978 & 59.1 & 40.9 & 100.0 \\
1979 & 61.5 & 38.5 & 100.0 \\
1980 & 60.3 & 39.7 & 100.0 \\
1981 & 49.0 & 51.0 & 100.0 \\
1990 & 46.5 & 53.5 & 100.0 \\
\hline
\end{tabular}

Fuente: Universidad de Chile (1957 a 1977), e Instituto Nacional de Estadísticas (INE) (1978 a 1990), último trimestre de cada año.

Este cambio en el servicio doméstico guarda relación con las transformaciones macroeconómicas y sociopolíticas experimentadas por el país entre 1973 y 1990. En 1974, junto con los cambios políticos y económicos iniciados por el gobierno militar que asumí el poder en 1973, se observó un aumento en la proporción de esas trabajadoras; lo mismo sucedió en 1981, coincidiendo con incrementos bruscos en los niveles de desempleo masculino. Parte del aumento de las tasas de participacion femenina en la metrópoli, y de la disminución proporcional de trabajadoras jovenes y solteras, correspondio a una mayor incorporación de mujeres casadas de los sectores populares al servicio doméstico no residente, como reacción al desempleo de los jefes de familia y a la caída de los ingresos familiares. Una vez que las mujeres urbanas casadas de los sectores populares aceptaron culturalmente la posibilidad de trabajar fuera del hogar -principalmente en el servicio doméstico- continuaron haciéndolo aun después de reducirse las tasas de desempleo masculino, posiblemente debido a la inestabilidad de los empleos y la precariedad de los ingresos de importantes segmentos de la población trabajadora.
Las tasas de participación de las mujeres fueron menores en los grupos de más bajos ingresos y de menor escolaridad en todo el período estudiado. El servicio doméstico era, hasta 1973, una ocupación desemper̃ada casi exclusivamente por mujeres migrantes que residian en la casa de sus patrones; en ella no participaban mujeres urbanas, especialmente si eran casadas. Sin embargo, en las coyunturas críticas -en términos de salarios y de empleo- del período 1970-1990 se registro un aumento sin precedentes de las empleadas domésticas no residentes (cuadro 1). En 1990, la mayoría de las mujeres en el servicio doméstico no vivían en la casa de sus patrones, tenían más de seis años de escolaridad $y$, en proporción elevada, estaban casadas.

Antes de las coyunturas recesivas de 1974 y 1981 las mujeres metropolitanas de los sectores populares no trabajaban después de casadas, pues cuidaban de sus hijos. A partir de esas coyunturas, una parte de ellas se incorporó al servicio doméstico no residente para sostener o ayudar a sostener a sus hijos, frente al desempleo masivo de los jefes de familia varones. Las propias normas de género - la valoración prioritaria de la maternidad- influyeron en la participación o falta de participación laboral entre las mujeres de escasos recursos. Su mayor o menor participacion parece haber dependido de la presencia de otros aportadores de ingresos en el hogar, y por esa vía, de los niveles de desempleo, remuneraciones y estabilidad de los trabajos masculinos.

El mercado de trabajo de Santiago en 1990 se caracterizaba por su segregación ocupacional por sexo. A pesar de que cerca del $40 \%$ de las mujeres económicamente activas estaban dedicadas a ocupaciones no manuales, éstas se concentraban en ocupaciones profesionales y técnicas de ingresos medios y bajos (más del 70\% eran maestras y enfermeras) y en los trabajos de oficina. Las trabajadoras manuales se situaban principalmente en los servicios personales y de los hogares y en unas pocas actividades industriales, especialmente la confección de ropa. Los varones en actividades no manuales se distribuían de manera más uniforme en diversos grupos, e inclúan directores y gerentes, y profesionales y técnicos de altos ingresos. Los trabajadores manuales se hallaban principalmente en las categorías de operarios y artesanos calificados, donde las mujeres estaban muy poco representadas (Muñoz, 1988; Szasz, 1994). Esta segregación ocurría a pesar de que los niveles de escolaridad de hombres y mujeres en Chile son semejantes, y a pesar de que las mujeres económicamente activas 
tienen una escolaridad más elevada que los hombres económicamente activos.

La segregación ocupacional se traduce en una notable diferencia entre los ingresos de los hombres y de las mujeres. Aunque para hacer una medición rigurosa de las diferencias de remuneraciones entre hombres y mujeres habria que cotejar los ingresos medios de cada ocupación con las horas trabajadas y con los niveles de escolaridad, pudimos aproximarnos a esa desigualdad estimando las remuneraciones medias de hombres y mujeres económicamente activos de la Región Metropolitana de Santiago en 1990 , considerando cada grupo ocupacional. De acuerdo con nuestras estimaciones, ${ }^{1}$ en 1990 las mujeres percibían en promedio ingresos por trabajo equivalentes al $62 \%$ de lo que ganaban los varones (cuadro 2). La desigualdad salarial parece especialmente marcada entre los trabajadores de los servicios personales y de los hogares, donde las mujeres ganan aproximadamente la mitad que los hombres en las mismas ocupaciones, aunque también es alta entre los obreros calificados, los vendedores y los oficinistas (Szasz, 1994). En el conjunto de América Latina, Chile aparecía en 1990 como uno de los países con mayor discriminación salarial por sexo (CEPAL, 1994), a pesar de la mayor escolaridad de las mujeres chilenas respecto del promedio de la región y de la ventaja escolar de las mujeres económicamente activas de Santiago respecto de los varones.

La diferencia proporcional entre los ingresos medios de hombres y mujeres económicamente activos hace de las mujeres un segmento en desventaja en el mercado de trabajo de Santiago. En 1990, la mitad de las mujeres trabajadoras percibían ingresos bajos, mientras que únicamente el $14 \%$ de los varones economicamente activos ganaban ingresos similares (cuadro 3 ).

CUADRO 2

\begin{tabular}{|c|c|c|c|}
\hline the & $\begin{array}{l}\text { Santiago de Cr } \\
\text { cada grupo oc } \\
\text { (Pesos chilenos d }\end{array}$ & $\begin{array}{l}\text { isual do I } \\
90 \\
\text { entajes) }\end{array}$ & ómicamente activas en \\
\hline Grupos ocupacionales & Hombres & Mujeres & $\begin{array}{l}\text { Ingreso femenino como } \\
\text { \% del ingreso masculino }\end{array}$ \\
\hline Directores y gerentes & 358469 & 315675 & 88.1 \\
\hline $\begin{array}{l}\text { Profesionales y técnicos } \\
\text { de altos ingresos }\end{array}$ & 281331 & 221522 & 78.5 \\
\hline $\begin{array}{l}\text { Profesionales y técnicos } \\
\text { de bajos ingresos }\end{array}$ & 110680 & 81638 & 73.8 \\
\hline Propietarios agrícolas & 115529 & 81491 & 70.5 \\
\hline Oficinistas & 92150 & 64440 & 69.9 \\
\hline Vendedores & .71855 & 45450 & 63.3 \\
\hline $\begin{array}{l}\text { Operarios y artesanos } \\
\text { calificados }\end{array}$ & 52602 & 32178 & 61.2 \\
\hline Obreros no calificados & 33910 & 32015 & 94.4 \\
\hline $\begin{array}{l}\text { Trabajadores de los } \\
\text { servicios personales y } \\
\text { de los hogares }\end{array}$ & 44274 & 23595 & 53.3 \\
\hline Total & 88862 & 54974 & 61.9 \\
\hline
\end{tabular}

Fuente: Elaboración propia a base de tabulados especiales del Instituto Nacional de Estadísticas. Basados en la Encuesta Nacional de Empleo, cuarto trimestre de 1990 (INE, 1978 a 1990 ).

- Comprende las profesiones cuyos ingresos medios mensuales en 1990 eran de 190000 pesos o más.

b Comprende las profesiones cuyos ingresos mensuales medios en 1990 eran inferiores a 190000 pesos.

\footnotetext{
1 Basadas en tabulaciones especiales de la Encuesta Nacional de
}

Empleo del Programa Integrado de Encuestas en Hogares. 

estratos de ingreso mensual y por eexo, 1990

(Pesos chilenos de 1990 y porcentajes)

\begin{tabular}{lrrr}
\hline $\begin{array}{l}\text { Estratos de ingreso } \\
\text { mensual medio } \\
\text { (pesos) }\end{array}$ & Hombres & Mujeres & Distribución porcentual \\
\hline Menos de 35000 & 14.3 & 51.3 & 28.3 \\
De 35000 a 76059 & 53.4 & 35.5 & 46.7 \\
De 76060 a 229999 & 21.4 & 9.6 & 16.9 \\
230000 o mas & 10.9 & 3.6 & 8.1 \\
Total & 100.0 & 100.0 & 100.0 \\
\hline
\end{tabular}

Fuente: Estimación propia sobre la base de tabulados especiales del Instituto Nacional de Estadisticas, obtenidos de la Encuesta Nacional de Empleo, cuarto trimestre de 1990 (INE, 1978 a 1990).

\section{V}

\section{Las inmigrantes recientes en el mercado laboral de Santiago}

La escasez de oportunidades laborales femeninas en los lugares de origen y la fuerte motivación laboral de las inmigrantes determino que su participacion fuese más elevada que la de las mujeres metropolitanas en el período 1960-1982. Esta diferencia se concentraba casi exclusivamente entre los 15 y los 24 años de edad, puesto que la participación de mujeres mayores de 24 años era semejante entre inmigrantes y nativas.

En la inmigración anterior a los años setenta, la proporción de mujeres menores de veinte anos era bastante elevada, aunque decreciente en el tiempo. Entre las inmigrantes del período 1977-1982, la proporción de adolescentes se redujo considerablemente. Si esta tendencia continúa, la participación económica de las inmigrantes se asemejará más a la de las mujeres metropolitanas.

Las diferencias en las tasas de desempleo por ocupación entre mujeres migrantes y no migrantes en 1982 sugieren que, con excepción de las jóvenes que ingresan al servicio doméstico, las inmigrantes tienen más dificultades para emplearse, especialmente si se trata de profesionales y técnicas, y que entre ellas es más frecuente el desempleo que entre las no migrantes. En el servicio doméstico ocurre lo contrario: el desempleo es menor entre las inmigrantes.

El análisis por grupos de ocupación indicó que entre 1960 y 1982 las inmigrantes se concentraron desproporcionadamente en las actividades manuales y en el servicio doméstico. Sin embargo, entre 1970 y 1982 su participación en el servicio doméstico decreció ligeramente, mientras que las no migrantes en este oficio aumentaron, como consecuencia del incremento antes descrito del servicio doméstico no residente.

La mayor concentración de mujeres inmigrantes en las actividades manuales se produce exclusivamente antes de los treinta años. En edades mayores, la distribución entre las actividades manuales y no manuales es semejante para ambos grupos de mujeres. Sin embargo, la desventaja de las inmigrantes jóvenes no se puede atribuír a su menor escolaridad. En 1970 la escolaridad de las inmigrantes y no inmigrantes era semejante, y en 1982 las inmigrantes superaban en escolaridad a las metropolitanas. En 1982, el 60\% de las inmigrantes económicamente activas se ubicaban en el servicio doméstico, mientras que $25 \%$ de las no inmigrantes se encontraban en ese grupo ocupacional. El $60 \%$ de las inmigrantes y el $\mathbf{4 0 \%}$ de las no inmigrantes que trabajaban como sirvientas tenían más de seis ańos de escolaridad, y más del $20 \%$ de las inmigrantes en ese tipo de empleos contaba con más de diez años de instrucción formal (Szasz, 1994). 
Las diferencias por grupos de edad y por escolaridad entre inmigrantes y no inmigrantes económicamente activas en 1982 eran menores. Sin embargo, ambos grupos de mujeres diferían notablemente en cuanto a su posición en el hogar. El $80 \%$ de las metropolitanas incorporadas al mercado de trabajo eran jefas de familia, esposas o hijas del jefe del hogar. En cambio apenas el $29 \%$ de las inmigrantes se encontraba en esa condición. La mayor parte no tenía lazos de parentesco en el hogar donde vivían. $\mathrm{El} 70 \%$ de las inmigrantes en el servicio doméstico eran mujeres sin hijos, mientras que el $61 \%$ de las mujeres metropolitanas en esa actividad tenía hijos.

Estas diferencias en la posición familiar constituyeron el único rasgo que explicaría las desventajas en la ocupación y los ingresos de las inmigrantes. Mientras las metropolitanas trabajaban para sostener o ayudar a sostener sus hogares, las inmigrantes carecían en su mayor parte de un hogar propio en la ciudad, y trabajaban para mantenerse y para tener un hogar de residencia.

Las diferencias culturales entre el género masculino y el femenino definen que los varones migrantes no experimenten la misma desventaja ocupacional que las mujeres migrantes. Entre 1962 y 1982, la estructura ocupacional y de ingresos de los inmigrantes varones en Santiago aparecía muy semejante a la de los metropolitanos, mientras que la mayor segregación de las mujeres inmigrantes hacia los servicios personales determinaba en ellas menores ingresos que las mujeres no inmigrantes (cuadro 4). En 1982 las inmigrantes ganaban, en promedio, cerca del $78 \%$ del ingreso promedio de las metropolitanas (Szasz, 1994).

CUADRO 4

Santiago de Chlie: Diatribuclón porcentual de la población econónlcamente actlva por estratos de Ingreso, por sexo y condlelón migratoria, en 1962 y 1982.

\begin{tabular}{|c|c|c|c|c|}
\hline \multirow[t]{2}{*}{$\begin{array}{l}\text { Estratos de } \\
\text { ingreso }\end{array}$} & \multicolumn{2}{|c|}{ Hombres } & \multicolumn{2}{|c|}{ Mujeres } \\
\hline & $\overline{\text { Inmigrantes }}$ & No migrantes & Inmigrantes & No migrantes \\
\hline $\begin{array}{l}\text { En } 1962 \\
\text { Menos del salario } \\
\text { mínimo }\end{array}$ & 8.9 & 6.2 & 45.3 & 23.4 \\
\hline $\begin{array}{l}\text { Entre el minimo } \\
\text { y el promedio }\end{array}$ & 40.8 & 36.0 & 39.4 & 40.6 \\
\hline $\begin{array}{l}\text { Entre el promedio } \\
\text { y } 3.5 \text { veces el } \\
\text { promedio }\end{array}$ & 40.5 & 45.1 & 14.6 & 32.7 \\
\hline $\begin{array}{l}\text { Más de } 3.5 \text { veces } \\
\text { el promedio }\end{array}$ & 9.8 & 12.7 & 0.7 & 3.3 \\
\hline $\begin{array}{l}\text { Total } \\
\text { En } 1982\end{array}$ & 100.0 & 100.0 & 100.0 & 100.0 \\
\hline $\begin{array}{l}\text { Menos del salario } \\
\text { mínimo }\end{array}$ & 19.1 & 18.1 & 61.6 & 36.6 \\
\hline $\begin{array}{l}\text { Entre el mínimo } \\
\text { y el promedio }\end{array}$ & 53.5 & 55.1 & 26.9 & 46.5 \\
\hline $\begin{array}{l}\text { Entre el promedio } \\
\text { y } 3 \text { veces el } \\
\text { promedio }\end{array}$ & 19.4 & 18.8 & 7.3 & 11.1 \\
\hline $\begin{array}{l}\text { Más de } 3 \text { veces } \\
\text { el promedio }\end{array}$ & 8.0 & 8.0 & 4.3 & 5.8 \\
\hline Total & 100,0 & 100.0 & 100.0 & 100.0 \\
\hline
\end{tabular}

Fuente: Para 1962: Encuesta sobre inmigración al Gran Santiago, CELADE, 1962 (Elizaga, 1970). Para 1982: Estimación propia basada en la distribución proporcional de la población inmigrante y no inmigrante por ocupación obtenida en la muestra del Censo de Población de 1982 , y los promedios de ingresos mensuales para hombres y mujeres en cada ocupación que seĩalan los resultados de la Encuesta Nacional de Empleo, cuarto trimestre de 1990 (INE, 1978 a 1990 ).

- Las cifras para 1962 se refieren a las inmigrantes llegadas entre 1952 y 1962 al Gran Santiago. Las cifras para 1982 se refieren a las llegadas a la Región Metropolitana entre 1977 y 1982. 
El único elemento que ayuda a comprender estas diferencias entre hombres y mujeres migrantes es la importancia del entorno familiar en el control social de la sexualidad femenina. La ausencia de familia no parece limitar las opciones ocupacionales de los varones, mientras que puede ser factor importante del elevado ingreso en el servicio domestico residente entre las inmigrantes más jóvenes.

\section{VI}

\section{Consideraciones finales}

El alto nivel de educación de las trabajadoras de Santiago de Chile en 1990 permite poner en tela de juicio la hipótesis de que algunos segmentos del mercado de trabajo están mal remunerados por su escasa calificación, y que ésta determinaría su baja productividad. Lo que sugiere más bien es una elevada demanda de mano de obra poco calificada para actividades de servicios personales en un contexto de concentración del ingreso, y escasas posibilidades de inserción ocupacional productiva y calificada para la mayoría de las mujeres educadas que se incorporan al mercado de trabajo.

En el horizonte de aquellas mujeres con 13 años o más de escolaridad - más de la mitad de las económicamente activas de Santiago- únicamente aparecen las ocupaciones de maestras, enfermeras, vendedoras y secretarias. Una minoría insignificante accede a puestos gerenciales o profesionales de ingresos elevados. Entre las trabajadoras manuales, a pesar de contar también con una escolaridad relativamente elevada respecto del conjunto de la región, las opciones se reducen principalmente al servicio doméstico considerablemente mayor que en el resto de América Latina-, la industria de confección de ropa y la venta ambulante. Más de la mitad de las mujeres economicamente activas perciben ingresos muy bajos, que solamente una minoria de hombres trabajadores perciben. Esto confirma que las mujeres son un segmento en desventaja en el mercado de trabajo de Santiago.

Para las mujeres que han inmigrado a la ciudad en años recientes, a pesar de contar con una escolaridad similar o más elevada que las no inmigrantes, las opciones ocupacionales son más reducidas, puesto que son segregadas mayoritariamente hacia los empleos manuales y el servicio doméstico. El mayor desfase entre calificación escolar, logros ocupacionales e ingresos que presentan las mujeres en general es aún más grave en el caso de las inmigrantes.

Condiciones de esta índole deben ser considera- das cuidadosamente al formular políticas sociales tendientes a reducir las inequidades en el mercado de trabajo. En un país como Chile, que ha mostrado solidez en los logros macroeconómicos, preocupa la mayor concentración de la riqueza y la heterogeneidad de ingresos laborales que ha generado el nuevo modelo de desarrollo.

Se han cifrado muchas esperanzas en la elevación de la productividad y de los ingresos de los segmentos vulnerables del mercado de trabajo a través de incrementos en su educación y capacitación laboral (Altimir, 1994; Rosenbluth, 1994; CEPAL, 1992). Sin embargo, los resultados de nuestro estudio sobre uno de esos segmentos del mercado de trabajo sugieren que los problemas de productividad e ingresos no se sitúan principalmente en carencias educacionales de la mano de obra, sino en las características de la demanda de fuerza de trabajo que el propio modelo genera. Entre esas características se incluye el limitado dinamismo de la demanda de mano de obra femenina escolarizada.

Las políticas sociales que busquen mejorar la equidad en los mercados de trabajo y disminuír los niveles de pobreza deben sopesar cuidadosamente la dimensión de género. Nuestros resultados sugieren que la segregación ocupacional y salarial que experimentan las mujeres trabajadoras de Santiago constituye una discriminación cultural y económica que acentúa el bajo aprovechamiento de la capacitación escolar de la mano de obra chilena. La mayor desventaja ocupacional y salarial que experimentan las inmigrantes se funda también en desigualdades de genero, puesto que la carencia de hogares y de redes de relaciones no afecta de igual manera a los inmigrantes varones.

Reducir las desigualdades de género en el acceso a los mercados de trabajo no puede ser únicamente una búsqueda al interior de los hogares, o en pequeños grupos de mujeres. La tarea abarca dimen- 
siones estructurales de las sociedades y definiciones centrales en las políticas públicas, que en el caso de Chile no están referidas a una marginación escolar de las mujeres, sino a componentes de género en la oferta y demanda de mano de obra en los mercados de trabajo.

\section{Bibliografia}

Altimir, O. (1994): Distribución del ingreso e incidencia de la pobreza a lo largo del ajuste. Revista de ta CEPAL $\mathrm{N}^{\circ} 52$, LC/G. 1824-P, Santiago de Chile, Comisión Económica pare América Latina y el Caribe (CEPAL), Naciones Unidas.

Aranda, X. (1982): Empleo, migración rural y estructura productiva agrícola, Contribuciones, № 9, Santiago de Chile, Facultad Latinoamericana de Ciencias Sociales (FLACSO).

Arriagada, I.(1990): Participación desigual de las mujeres en el mundo del trabajo, Revista de la CEPAL, No $\mathbf{N}^{\circ}$, LCG. 1613-P. Santiago de Chile, CEPAL, Naciones Unidas.

CEPAL (1992): Equidad y transformacion productiva: un enfoque integrado, LC/G.1701/Rev.1-P, Santiago de Chile. Publicación de las Naciones Unidas, $\mathrm{N}^{\circ}$ de venta S.92.IIG.5.

(1994): Mujer y trabajo urbano en América Latina: el significado de los cambios en los años noventa, Documentos presentados a la Sexta Conferencia Regional sobre la Integración de la Mujer en el Desarrollo Económico y Sacial de America Latina y el Caribe, LCL. 844 (CRM.6/6)/Rev. 1 , Mar del Plata, Argentina, 26 al 30 de septiembre, Naciones Unidas.

De Oliveira, 0. (1984): Migración femenina, organización familiar y mercados laborales en México, Comercio exterior, vol. 34 $N^{\circ}$ 7, México D.F., Banco Nacional de Comercio Exterior. S.A., julio.

Elizaga, J. (1970): Migraciones a las áreas metropolitanas de Amé rica Latina, Santiago de Chile, Centro Latinoamericano de Demografía (CELADE).

Elton, Ch. (1978): Migracion femenina en América Latina: factores determinantes, Santiago de Chile, Centro Latinoamericano de Demograffa (CELADE).

García, N. (1991): Reestructuracion, ahorro y mercado de trabajo, Investigaciones sobre empleo, $\mathrm{N}^{\circ}$ 34, Ginebra, Programa Regional del Empleo para América Latina y el Caribe (PREALC), Organización Internacional del Trabajo (OIT).

(1993): Ajuste, reformas y mercado laboral: Costa Rica (1980-J990), Chile (1973-1992), Mexico (1981-1991), Ginebra, PREALC, OrT.

Garcfa de Fanelli, A.M. (1989): Patrones de desigualdad social en la sociedad modema: una revisión de la literatura sobre discriminación ocupacional y salarial por género, Desarrollo económico, vol. $29, \mathrm{~N}^{\circ} 114$, Buenos Aires, Instituto de Desarrollo Economico y Social (IDES).

Herold, J. (1979): Female migration in Chile: Types of moves and socioconomic characteristics, Demography, vol. $16, \mathrm{~N}^{\circ} 2$ Washington, D.C., Population Association of America.

Hugo, G. (1991): Migrant women in developing countries, United Nations Expert Group Meeting on Feminization of Intemal Mi- gration, Aguascalientes, México, 22 al 25 de octubre, mimeo. INE (Instituto Nacional de Estadísticas) (1978 a 1990): Encuesto nacional de empleo, Programa Integrado de Encuestas en Hogares, Santiago de Chile.

Lattes, A. (1989): Emerging patterns of territorial mobility in Latin America: Challenges for research and action, trabajo presentado a la International Population Conference (Nueva Delhi) Lieja, Bélgica, Unión Internacional para el Estudio Científico de la Población (UIECP), mimeo.

Lim, L. (1989): Processes shaping intemational migration flows, trabajo presentado a ia International Population Conference, (Nueva Delhi), Lieja, Bélgica, UIECP, mimeo.

Martínez, J. (1990); Patrones migratorios interregionales en Chile: analisis de casos seleccionados, serie $\mathrm{A}, \mathrm{N}^{\circ} 212$, LCDEM/G. 100, Santiago de Chile, CELADE.

Muñoz, A. (1988): Fuerza de trabajo femenina: evolución y tendencias, Mundo de mujer: continuidad y cambio, Santiago de Chile, CEM (Centro de Estudios de la Mujer).

Pardo, L. (1987): Participación de las mujeres en la fuerza de trabajo: tendencias y características, 1a. parte, Revista economía y administración. $\mathrm{N}^{\circ} 61$, Santiago de Chile, Universidad de Chile, Facultad de Ciencias Economicas y Administratjvas.

Pollack, M. (1990): Women workers and the economic cycle, trabajo presentado a la Conference on Weathering Economic Crisis: Women's Economic Responses to Recession in Latin America and the Caribbean (CEPAL, Santiago de Chile, 27 al 30 de mayo), mimeo.

PREALC (1979): Expectativas migratorias de la joventud campesi$n a$, Documento de trabajo, $N^{\circ} 178$, Ginebra, olT. (1991): Empleo y equidad: desaffo de los noventa, Santiago de Chile, orr.

Raczynski, D. y P. Vergara (1979): Condicionantes del comportamiento migratorio de Las areas rurales en Chile, Santiago de Chile, Corporación de Investigaciones Económicas para Latinoamerica (CIEPLAN).

Recchini de Lattes, Z. (1990): La mujer en la migración interna e internacional con especial referencia a América Latina, Bolethn de población de las Naciones Unidas, $N^{\circ} 27$, Nueva York.

Rosenbluth, G. (1994): Informalidad y pobreza en America Latina, Revista de la CEPAL, No 52, LC/G. 1824-P, Santiago de Chile, CEPAL, Naciones Unidas.

Szasz, I. (1994): Mujeres inmigrantes y mercado de trabajo en Santiago, serie E, $\mathbf{N}^{\circ} 39$, Santiago de Chile, CELADE.

Universidad de Chile (1957 a 1977): Encuesta nacional de ocupación y desocupación del Gran Santiago, Santiago de Chile, Departamento de Economía. 